\title{
Kommunikative Kirche angesichts des dritten Jahrtausends
}

\author{
von Walbert Bühlmann
}

Vom Missionsbefehl Christi bis zum neuesten und klassischen Dokument „Evangelii nuntiandi“ über Evangelisierung in der Welt von heute (8.12.1975) weiß sich die Kirche demselben Auftrag verpflichtet: der Menschheit Shalom auszurufen, Gottes Gute Nachricht $z u$ verkündigen, umfassendes Heil $z u$ verheißen, und dies ohne jegliche Einschränkung weder der Zeit noch des Ortes, also bis an die Grenzen der Erde und bis an das Ende der Zeiten, also auch bis ins kommende dritte Jahrtausend hinein.

Die Art und Weise, diesen Auftrag zu erfüllen, wechselt freilich mit dem je wechselnden geographischen und sozialen Rahmen der zu evangelisierenden Menschheit und mit den sich entwickelnden technischen Möglichkeiten der Verkündigung. Nun, bei allem Schlechten, das Kirchenleute gern an der heutigen Zeit sehen, glaube ich, würden die Apostel damals frohlockt haben, hätten sie die gewaltigen technischen Möglichkeiten der Verkündigung in der heutigen Zeit voraussehen können. Wir gehen ohne Zweifel zwar nicht einer idealen Welt, wohl aber einem Höhepunkt der Verkündigung des Evangeliums entgegen. Wir stehen jedenfalls vor globalen Möglichkeiten und damit vor einer globalen Herausforderung.

\section{Die kommunikationshungrige Welt}

Der Mensch ist von Natur aus wißbegierig. Aber der Intensitätsgrad dieser Begier und der quantitative Umfang des tatsächlichen Wissens schwanken sehr von einer Menschengruppe, die noch zufrieden in ihrem Clan lebt und von der das Sprichwort gilt: „Was man nicht weiß, macht einen nicht heiß", bis zum modernen Menschen, der mit der gesamten Welt verbunden ist und darum alles wissen, alles haben möchte. Eine der entscheidenden Leistungen, welche die Welt zur "neuen Welt" machten, war deren verkehrs- und nachrichtentechnische Erschließung. Als Christoph Columbus am 12. Oktober 1492 Amerika entdeckte, erfuhr man es in Europa, d.h. am spanischen Hof, erst fünf Monate später. Als am 14. April 1865 der amerikanische Präsident Lincoln ermordet wurde, erhielt man in Europa die Kunde davon erst 12 Tage hernach.

Und heute? Was vor wenigen Jahrzehnten in den phantasievollen Romanen eines Jules Verne hätte stehen können, ist zur technischen Realität geworden. Wenn irgendwo in der Welt etwas von Bedeutung geschieht, hört man es abends in allen Stuben am Funk, liest man es anderntags in allen Zeitungen. Ja, wichtige Ereignisse, Papstbesuche im Heiligen Land oder vor den Vereinten Nationen, Sportanlässe, politische Ereignisse, können mit keiner Sekunde Verspätung in aller Welt vor dem Bildschirm verfolgt werden. Diesbezüglich sind wir also nicht bloß an der Schall-

Prof. Dr. Walbert Bühlmann OFMCap. ist Leiter des Generalsekretariates für die Kapuzinermissionen in Rom, Missiologe und Autor des Missions-Reports: "Wo der Glaube lebt ${ }^{\text {. }}$. 
mauer, die noch durchbrochen werden kann, sondern an der absoluten Grenze einer Entwicklung angelangt.

Die Kirche als das erste Welt-Unternehmen, der das "Gehen in alle Welt" und das "Verkünden an alle Geschöpfe" in die Grundkonstitution eingeschrieben steht, muß im höchsten Maß daran interessiert sein, ob ihre Boten monatelang und mühsam über die Meere fahren oder über Nacht im Flugzeug an ihre Posten gebracht werden; ob ihre Botschaft nur allmählich im Lauf der Jahrhunderte die verschiedenen Länder der Erde erreicht - oder im selben Augenblick überallhin ausgestrahlt werden kann.

Das Volumen der Wißbegier der heutigen Menschen ist also unerhört gewachsen. Es wird freilich nie an einer absoluten Grenze ankommen, denn der Mensch ist auf Unendliches angelegt. Auch das Volumen der wißbegierigen Menschen steht gegenwärtig in einer einzigartigen Entwicklungsphase der Geschichte. Es brauchte Jahrhunderttausende oder Jahrmillionen, um die Menschen durch Kriege, Epidemien und Naturkatastrophen hindurch 1970 auf 3,6 Milliarden anwachsen zu lassen. Es dauert aber gegenwärtig nur 30 Jahre, um aus dieser Zahl das Doppelte, nämlich 6,5 Milliarden im Jahr 2000 werden zu lassen. Mit den neuen Möglichkeiten der Geburtenkontrolle wird diese Voraussicht wohl etwas reduziert werden. Trotzdem: die Kirche steht vor einer Masse Menschen wie noch nie in der Geschichte und müßte an ihrer Aufgabe fast verzweifeln, wenn ihr nicht in einer providenziellen Synchronisierung ausgerechnet in dieser Zeit der auftretenden Menschenmasse die technische Möglichkeit der Massenmedien angeboten würde, mit der sie diese Masse, selbst wenn sie noch viel größer würde, erreichen kann.

Man weiß nun freilich, daß Kommunikation nicht ankommt, wenn sie nicht anspricht. Das Ansprechen aber hängt nicht bloß von der guten oder schlechten Gestaltung, nicht einmal bloß vom Inhalt $a b$, sondern vor allem vom bereits vorhandenen Interesse. Wenn das fehlt, fließt die beste Kommunikation ab wie ein Platzregen über die harte Erde. Wird also die Menschheit im Jahr 2000 noch interessiert sein an religiöser Kommunikation?

Die Marxisten und viele andere beantworten die Frage eindeutig negativ. Für sie wird bis dann der Prozeß der Entmythologisierung, der Säkularisierung, der Zerstörung der religiösen Gewohnheiten und Strukturen sein Ziel erreicht haben. Ihre Erwartung wird einem fast mit mathematischer Präzision glaubhaft gemacht: Vor 100 Jahren hätten noch alle geglaubt, heute glaube noch die knappe Hälfte, im Jahr 2000 würde niemand mehr glauben außer einigen bornierten Individuen.

Dieser Prognose gegenüber möchte ich die These aufstellen, daß die Menschen im Jahr 2000 nicht bloß kommunikationshungrig, sondern auch heilshungrig sein werden, und zwar mehr denn je. Genau, weil sie nicht bloß in der westlichen, sondern auch in der Dritten Welt aus den religiösen Strukturen, in denen sie sich bisher gesichert fühlten, die ihnen gar keinen ernstlichen religiösen Zweifel aufkommen ließen, herausgefallen sind, sich plötzlich auf sich selbst verwiesen sehen und damit zu religiös Suchenden und Fragenden werden. Selbst wenn der Mensch möchte, er kann die letzte Sinnfrage seines Lebens nicht von sich abschütteln. Sie steckt tief in ihm wie seine eigene Natur. Was ihm übrigens das Leben bietet, ist eine lange Reihe von nie vollen Freuden, von nie ganz erreichten Zielen, von immer rätselhaft bleibenden Schmerzen und Todesgedanken, und als Folge daraus eine innere Leere und Frustration, oder eine sich selbst auferzwungene Härte mit dem Mut zum Dasein, 
oder schließlich die religiöse Antwort, die im Grunde eben doch nicht bloß trostvoller, sondern auch menschengemäßer ist.

Der moderne Mensch wird vermutlich scharenweise aus den angestammten Kirchen und Religionen auswandern. Aber er findet damit nicht ohne weiteres eine bessere Heimat. Er wird vielmehr zum Irrenden, Fragenden, zum vom Heimweh Getriebenen, man könnte sagen, zum religiösen Nomaden. Das dritte Jahrtausend wird darum nicht das Jahrtausend der religionslosen Menschen sein, wohl aber das Jahrtausend der religiösen Nomaden, die „interessierter“ sein werden an religiöser Botschaft als ihre religiös seßhaften Vorgänger.

\section{Die kommunikations-beauftragten Kirchen}

Wenn eine Institution sich um Kommunikation in allen möglichen Formen kümmern soll, dann die Kirche. Wie der Mensch von Natur aus kommunikations- und heilshungrig ist, so ist die Kirche von Natur aus zur Kommunikation gesandt, mehr, sie ist Kommunikation. Man müßte hier das erste Kapitel von "Ad Gentes“ und ebenso das erste Kapitel von „Evangelii nuntiandi“ ${ }^{\text {“ }}$ wiedergeben, um zu zeigen, wie vom Vater zum menschgewordenen Sohn, zum gesandten Geist, zur gegründeten Kirche eine vorgegebene, irreversible Dynamik verläuft, wie Kommunikation, Koinonia, Kommunion Urbegriffe für das innertrinitarische Leben sind und es darum auch für die Gemeinschaften der ersten Christen wurden. Am Anfang war das Wort. In der Mitte der Geschichte, „als die Zeiten erfüllt waren“, ist das Wort Mensch geworden. Im bleibenden Rest der Geschichte soll es unser gemeinsames Anliegen sein, „daß das Wort des Herrn sich raschen Laufes verbreite und verherrlicht werde" (2 Thess 3,1$)$.

Dem "Wort des Herrn" hat sich in späteren Jahrhunderten wie durch eine natürliche Entwicklung auch das „Wort der Menschen“ hinzugefügt. Die Kirche war lange Zeit die hauptsächlichste Vermittlerin von Wissen und Wissenschaft, von religiöser und profaner Schulung. Nicht umsonst ist in manchen Missionssprachen "lesen" synonym geworden mit "beten": jene, die in den Schulen lesen lernten, waren dieselben, die am Sonntag gemeinsam beteten.

Heute ist das anders geworden. Die profane - wenn sie nur profan wäre! - und ideologisch geprägte Welt hat die Kommunikation beschlagnahmt. Die Kommuni-

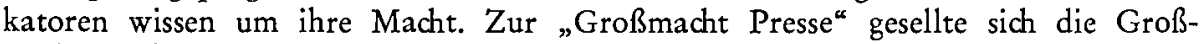
macht Funk und Fernsehen. Die Marxisten mit ihrer eigenen Teclunik der Sensibilisierung setzen in ihren Staaten vom Morgen bis zum Abend die Augen und Ohren ihrer Bürger dem Einfluß von Slogans und von Informationen aus. Nicht umsonst helfen sie auch den jungen Staaten, wo sie Fuß zu fassen hoffen, immer zuerst mit guten Sendeanlagen. Sie wissen: Wer die Kommunikation in der Hand und 2000 Soldaten zur Verfügung hat, beherrscht ein Land. Wer diese Mittel heute gebraucht, wird zum Lehrer der Welt. Wer sie nicht gebraucht, wird mitsamt seiner Gruppe zu den "Analphabeten“ von morgen gehören, die zwar lesen und schreiben können, aber mit den wichtigeren Mitteln des Hörens und Sehens durch die Massenmedien nicht umzugehen verstehen. Früher wurde das Kind in der Familie, in der Schule, in der Kirche erzogen und geformt, heute von der öffentlichen Meinung im "Weltdorf“. Wer diese öffentliche Meinung beherrscht, herrscht weitgehend über die Menschen. 
Daß dies nicht bloß in den entwickelten Staaten des Westens und Ostens geschieht, sondern daß auch in der südlichen Welt die Transistoren im hintersten Dorf zu finden sind, daß schon in absehbarer Zeit auch TV-Transistoren auf den Markt geworfen werden, löst einen ungeheuren Kulturschock aus, indem es jene Völker fast innerhalb einer Generation von der traditionellen, abgeschlossenen Gesellschaft in den Betrieb der modernen Welt hineinreißt.

Es schafft aber auch für die Kirche eine Chance einzigartigen Ausmaßes. Sie kann nun die Lautstärke und die Reichweite ihres Wortes tausendfach verstärken. Sie kann von der Mikro- zur Makroevangelisierung schreiten, die Botschaft nicht mehr bloß den einzelnen und dem Dorf, sondern der Weltgemeinschaft verkünden. Um die ganze Erde liegt bereits ein Netz von Strahlungszentren, die sich ständig vermehren und vervollkommnen, die auch das Evangelium in Ton und Bild in den hintersten Winkel der Erde tragen können. Falls wir Anschluß an diese technischen Errungenschaften finden, sind wir in der Lage, die Welt im wahrsten Sinn in einen Hörraum des Evangeliums zu verwandeln.

Jetzt verstehen wir die ernsten Worte, die Paul VI. in „Evangelii nuntiandi“ (n. 45) im Blick auf die Massenmedien sagt: Die Kirche müßte sich schuldig fühlen vor ihrem Herrn, würde sie diese mächtigen Mittel nicht gebrauchen; sie seien die moderne und wirksame Form der Kanzel, eine Herausforderung, die man nützen müsse, die freilich den persönlichen Kontakt von Mensch zu Mensch nie überflüssig mache.

Die Kirche wird sich heute nicht mehr zumuten, hauptsächlichster InformationsTräger zu sein. Aber sie darf nie davon abstehen, komplementär an diese Masse von Kommunikation ihren spezifischen und sehr wichtigen Beitrag zu leisten. Sie hat vor allem das Wort des Heils und der Hoffnung in diese Welt hineinzusagen, den jungen Menschen, die überall in der Welt voller Hoffnung sind, zu bestätigen, daß diese Hoffnung berechtigt sei, weil Gott es in der Tat gut mit ihnen meine; den Erwachsenen und Verantwortlichen Mut zu machen, Impulse zu geben, alles zu tun, damit Gerechtigkeit und Friede herrschen und die Menschen nicht zu sehr an ihren Hoffnungen scheitern müssen; den Kranken und Alten das Vertrauen in die $\mathrm{Zu}$ kunft zu erhalten, da der Christ ja nicht bloß Hoffnung in dieser Welt, sondern über diese Welt hinaus zu haben berechtigt ist. Noch eine andere komplementäre Aufgabe kann die Kirche erfüllen: Man stellt fest, daß bei allem Riesenangebor von Kommunikation die Menschen immer einsamer werden; sie konsumieren die Kommunikation als Individuen, aber nicht als Gemeinschaft, selbst wenn sie es in Gemeinschaft tun. Die Kirche, ihrem Wesen nach Koinonia, Kommunion, Gemeinschaft, muß hier in die Bresche springen und dem modernen heim- und heimatlosen Einzelmenschen wieder Geborgenheit und Gemeinschaft schenken.

Nachdem in den Entwicklungsländern nach der Euphorie der Unabhängigkeitsfeiern und der vielen Entwicklungspläne eine große Ernüchterung eingetreten ist; nachdem wir im Traum vom unbegrenzten Fortschritt von der brutalen Realität der begrenzten Mittel aufgeschreckt wurden und überhaupt erkannt haben, daß der „American way of life" nicht unbedingt als Ideal gelten kann; nachdem die verbissene Art der Marxisten, die Welt zu verändern, bisher noch nirgendwo volle Freude auszulösen vermochte und der freie Mensch jedenfalls darin keine bessere Alternative erkennen kann, bleibt der Kirche mehr als je die Aufgabe, diesen Menschen den Mut, die Zuversicht, das Lächeln zu erhalten. Angesichts der tiefgreifenden Glaubens-, Hoff- 
nungs- und Liebesprobleme der gegenwärtigen Menschheit wäre es für die Kirche beschämend, sich wegen kleiner ritueller, disziplinarischer, theologischer Einzelfragen zu zerstreiten. Wozu Gott sie brauchen will, das ist, dieser Menschheit immer wieder und glaubwürdig zu sagen, daß es einen guten Gott gibt, daß seit Ostern auch aus den schlimmsten Situationen Auswege offenstehen, daß die Christen durch ihr Leben bereits etwas vom kommenden Gottesreich in dieser Welt sichtbar machen sollen.

In dieser grundlegenden Aufgabe können die Kirchen nicht mehr nebeneinander ihre Wege gehen oder gar gegeneinander mit ihrer Hoffnung hausieren. Wenn sich auf einem Gebiet Okumenismus gebieterisch aufdrängt, dann in der Begegnung mit der Weltmenschheit. In den Massenmedien (Buchläden, Bibelverbreitung, Funk, Fernsehen) sollen wir nur gemeinsam Zeugnis für den einen Christus ablegen. Die Unterscheidungslehren sollen auf den internen Religionsunterricht beschränkt bleiben. Der Ather soll nicht verkonfessionalisiert, er soll verchristlicht werden, erfüllt werden mit der Botschaft von der Erlösung, wie damals der Himmel von Bethlehem.

In dieser Hinsicht sind in den letzten Jahren ohne Zweifel viele gute Fortschritte gemacht worden. Denken wir an die gemeinsamen Absprachen für die christlichen Sendungen in vielen nationalen Radio-Programmen der westlichen und der südlichen Welt - oder an die ökumenische Bibelarbeit. 1961 wurde sehr behutsam eine erste Initiative einer gemeinsamen Bibel in Afrika unternommen, nämlich für die Suaheli-Sprache. Gegenwärtig aber laufen in Afrika 78 interkonfessionelle Projekte für Bibelübersetzung und -verbreitung.

Ein neuer akuter Fall, der uns aus Not vielleicht einen kühnen Schritt in ökumenischer Zusammenarbeit machen lassen könnte: Nachdem die äthiopische Regierung den lutherischen Sender "Stimme des Evangeliums" in Addis Abeba verstaatlicht hat und diese großartige Stimme, die fast ganz Afrika und den Nahen Osten mit christlicher Freude und Hoffnung erfüllte, verstummen mußte, könnte es da nicht denkbar sein, daß ein Teil jenes Geldes und des tüchtigen Personals in Radio Vatikan investiert würde, dem eine Blutauffrischung nur gut tun würde?

Um die Chancen der kirchlichen Kommunikation im Jahr 2000 einigermaßen auszunützen, müßten jetzt schon viele mutige Schritte unternommen werden. Auf dem Gebiet der katholischen Massenmedien in der Dritten Welt bekommt man den Eindruck, daß überall guter Wille vorhanden ist, daß an vielen Orten gute Ansatzpunkte vorliegen, daß es aber noch an großzügiger Planung und Zusammenarbeit und am entscheidenden Durchstoß fehlt. Für das riesige Land Indien, wo die Kirche genau den Auftrag hätte, nicht bloß an ihre 10 Millionen Katholiken zu denken, sondern allen 650 Millionen Indern etwas zu bieten, bemerkt ein Kenner der Lage, P. E. Zeitler SVD: „Was den Einsatz der Kirche in Indien und ihre Missionsmethode betrifft, so wird man feststellen müssen, daß man nicht über die zu Beginn des 20. Jahrhunderts erfolgreichen Methoden hinausgekommen ist, obwohl wir heute in eine völlig neue Welt eingetreten sind, die $\mathrm{zu}$ einem wesentlichen Teil von den Massenmedien bestimmt wird, deren Bedeutung die indische Kirche noch nicht erkannt hat." Dasselbe stellten die 30 Vertreter aus 12 afrikanischen Ländern fest, die kürzlich in Nairobi zu einem Seminar über „Audiovision und Evangelisierung “ zusammengekommen waren. Sie meinten, es sei unterdessen noch zu keinem konsequenten, bewußten und praktizierten Brüdkenschlag zwischen medienoffenem Volk und botschafttragender Kirche gekommen. 
Was der enthusiastische und prophetische John Mott (1865-1955) in der Anglikanischen Kirche und von allen Christen unermüdlich forderte: „Evangelisierung der Welt in dieser Generation", ist zur konkreten Aufgabe geworden. Es liegt in unserer Hand, allen Menschen die Botschaft des Evangeliums bekannt zu machen. Und da gemäß „Evangelii nuntiandi“ alle Evangelisierten ihrerseits Evangelisatoren werden sollen, könnte man die Hoffnung haben, daß die über die Massenmedien von der christlichen Botschaft Berührten im persönlichen Kontakt mit den Christen, vor allem mit kleinen Gruppen echter Christen, weitere Gelegenheit haben, die Botschaft und ihre Träger kennenzulernen und sich dazu ihre Gedanken zu machen. Wir dürfen auf jeden Fall gewiß sein, daß das Ausstreuen der Botschaft nie wirkungslos sein wird. Selbst wenn man es nicht kontrollieren kann, der Same wird keimen und sprießen, von selbst Frucht tragen, und früher oder später werden wir plötzlich sagen können: Die Ernte ist da! (Mk 4, 26-29).

Ob aus der Evangelisierung dann als Schlußergebnis die Christianisierung erfolge, der offizielle Eintritt des Evangelisierten in die Gemeinschaft der Christen, das liegt nicht mehr in unserer Hand. Da stehen wir vor dem Geheimnis der Freiheit des Menschen und der Freiheit Gottes. Ob der „Ratschluß Gottes, alles im Himmel und auf Erden in Christus als dem Haupte zusammenzufassen" (Eph 1,10), der in der jetzigen „Fülle der Zeit ausgeführt ist“, sich auch sichtbar jetzt, innergeschichtlich oder erst eschatologisch verwirkliche, darüber ist uns nichts geoffenbart. Daß es aber grundsätzlich bereits geschehen ist und einmal auch offenkundig erscheinen wird in der Parusie des Herrn, das ist für uns Antrieb genug, jetzt schon mit allen Mitteln, auf alle Weisen den Menschen zu verkünden, was an ihnen geschehen ist, daß ihnen Friede, Heil zugesprochen ist, da Gott sie alle liebt.

\section{S U M M A R Y}

It is likely that modern men will leave the traditional churches in large numbers, but this does not imply that they will feel at home elsewhere. Man will become alienated, questioning everything. The third millenium will not be the millenium of man without religion, but of man as a religious nomad. The Church, basically an instrument of communication, has the obligation of announcing the message of a good God and the possibility of man's overcoming even the most difficult situation since the happening of Easter. Christian living must bear witness to the coming of the Kingdom of God. This task is to be undertaken by all Christian Churches working together. Christians should speak with one voice. In the Third World particularly there is good will, but overall planning and co-ordination is still missing.

\section{RESUMÉ}

L'homme moderne émigrera vraisemblablement en masse des églises et des religions héréditaires, mais ne trouvera pas tout simplement pour cela une meilleure patrie. Il deviendra plutôt un errant, un questionneur. Le troisième millénaire ne sera pas celui de l'homme sans religion, mais du nomade religieux. L'Eglise, en tant qu'institution communicative, a ici le devoir d'annoncer à l'aide de tous les moyens modernes qu'il n'y a qu'un bon Dieu, que, depuis Pâques, il y a des issues pour sortir des situations mêmes les plus délicates et que les chrétiens doivent, par leur vie, rendre déjà un peu visible le règne de Dieu à venir. Ce devoir 
doit être rempli par toutes les Eglises chrétiennes, en commun, de façon oecuménique. Les chrétiens doivent parler d'une seule voix. La bonne volonté est bien partout présente dans le tiers-monde, mais il manque encore de la planification généreuse et de la collaboration.

\section{RE S U M E N}

El hombre moderno emigrará probablemente en grupos de su Iglesia ó de su religión, pero no encontrará sin más una patria mejor. Se convertirá por el contrario en nómada, en interpelador. El tercer milenio no será el de los hombres sin religión, sino el de los nómadas religiosos. La Iglesia, como institución comunicadora, tiene la misión de anunciar por todos los medios modernos que existe un Dios bueno, que desde la Resurrección también quedan caminos abiertos hasta para las peores situaciones y que los cristianos deben dejar entrever con su vida algo del Reino de Dios venidero. Esta misión debe ser cumplida por todas las iglesias conjunta, ecuménicamente. Los cristianos deben hablar con una sola voz. En el Tercer Mundo existe ciertamente buena voluntad por doquier pero falta todavía mucho en la planificación y coordinación. 